\title{
Impact of order set design on urine culturing practices at an academic medical centre emergency department
}

\author{
Satish Munigala, ${ }^{1}$ Ronald R Jackups, ${ }^{2}$ Robert F Poirier, ${ }^{3}$ Stephen Y Liang, ${ }^{1}$ \\ Helen Wood, ${ }^{4}$ S Reza Jafarzadeh, ${ }^{5}$ David K Warren ${ }^{1}$
}

${ }^{1}$ Department of Internal Medicine, Washington University in St. Louis, St. Louis, Missouri, USA

${ }^{2}$ Department of Pathology and Immunology, Washington University in St. Louis, St. Louis, Missouri, USA

${ }^{3}$ Emergency Medicine, Washington University in St. Louis, St. Louis, Missouri, USA ${ }^{4}$ Hospital Epidemiology and Infection Prevention, BarnesJewish Hospital, St. Louis, Missouri, USA

${ }^{5}$ Clinical Epidemiology Research and Training Unit, Boston University, Boston, Massachusetts, USA

\section{Correspondence to} Professor David K Warren, Department of Internal Medicine Washington University in St. Louis, St. Louis, Missouri, USA; dwarren@wustl.edu

Received 12 May 2017 Revised 6 December 2017 Accepted 15 December 2017 Published Online First 20 January 2018

\section{SLinked}

- http://dx.doi.org/10.1136/ bmjqs-2017-007578

\section{Check for updates}

To cite: Munigala $S$, Jackups RR, Poirier RF, et al. BMJ Qual Saf

2018;27:587-592.

\begin{abstract}
Background Urinalysis and urine culture are commonly ordered tests in the emergency department (ED). We evaluated the impact of removal of order sets from the 'frequently ordered test' in the computerised physician order entry system (CPOE) on urine testing practices. Methods We conducted a before ( 1 September to 20 October 2015) and after (21 October to 30 November 2015) study of ED patients. The intervention consisted of retaining 'urinalysis with reflex to microscopy' as the only urine test in a highly accessible list of frequently ordered tests in the CPOE system. All other urine tests required use of additional order screens via additional mouse clicks. The frequency of urine testing before and after the intervention was compared, adjusting for temporal trends.

Results During the study period, 6499 (28.2\%) of 22948 ED patients had $\geq 1$ urine test ordered. Urine testing rates for all ED patients decreased in the post intervention period for urinalysis (291.5 pre intervention vs 278.4 per 1000 ED visits post intervention, $\mathrm{P}=0.03$ ), urine microscopy (196.5vs179.5, $\mathrm{P}=0.001$ ) and urine culture (54.3vs29.7, $\mathrm{P}<0.001)$. When adjusted for temporal trends, the daily culture rate per $1000 \mathrm{ED}$ visits decreased by $46.6 \%(-46.6 \%, 95 \% \mathrm{Cl}-66.2 \%$ to $-15.6 \%)$, but urinalysis $(0.4 \%, 95 \% \mathrm{Cl}-30.1$ to $44.4 \%)$, microscopy $(-6.5 \%, 95 \% \mathrm{Cl}-36.0 \%$ to $36.6 \%)$ and catheterised urine culture rates $(17.9 \%, 95 \% \mathrm{Cl}-16.9$ to 67.4) were unchanged.

Conclusions A simple intervention of retaining only 'urinalysis with reflex to microscopy' and removing all other urine tests from the 'frequently ordered' window of the ED electronic order set decreased urine cultures ordered by $46.6 \%$ after accounting for temporal trends. Given the injudicious use of antimicrobial therapy for asymptomatic bacteriuria, findings from our study suggest that proper design of electronic order sets plays a vital role in reducing excessive ordering of urine cultures.
\end{abstract}

\section{INTRODUCTION}

Urinalysis and urine culture are commonly ordered tests in the emergency department (ED). ${ }^{1}$ However, the diagnostic yield of urine culture for patients with undifferentiated abdominal pain is low and urine cultures are not recommended for evaluation of uncomplicated urinary tract infections (UTI). ${ }^{1}$ Urine cultures performed without clear clinical indications increase healthcare costs and contribute to the unnecessary treatment of asymptomatic bacteriuria (ASB). ${ }^{1}{ }^{2}$ Despite several recommendations, antimicrobial therapy directed against ASB remains common in clinical practice and may promote antimicrobial resistance. ${ }^{3}$

Previously examined interventions to reduce unnecessary urine culture tests have included automatic cancellation of urine cultures for specimens with low-risk urinalysis, ${ }^{1}$ two-step urine culture ordering $^{4}$ and antimicrobial stewardship efforts. ${ }^{5}$ Jones $e t a l^{1}$ developed a reflex urine culture cancellation protocol in the ED based on low-risk findings (negative nitrites, negative leukocyte esterase, negative bacteria and $\leq 10$ white blood cell (WBC) per high-power field) for infection on urinalysis and concluded that using these criteria would have eliminated $39 \%$ of unnecessary cultures. Stagg et al implemented a two-step model for urine culture ordering in the ED which resulted in decrease in urine cultures processed, a decrease in callbacks for positive results and an improvement in antimicrobial prescribing for UTI. Other quality improvement projects include antimicrobial stewardship efforts where antibiotic recommendations were made based on the culture results. ${ }^{5}$ However, there are limited data on design and location of the urine culture order sets and its role in ED urine testing practices.

In this study, we retained only 'urinalysis with reflex to microscopy' and removed all other urine orders from the 
Table 1 Urine order set definitions

\begin{tabular}{ll}
\hline Urine order set & Definitions \\
\hline $\begin{array}{l}\text { Urinalysis with reflex to } \\
\text { microscopy }\end{array}$ & $\begin{array}{l}\text { Perform urine dipstick, if positive for } \\
\text { protein>trace, any blood then reflex to } \\
\text { microscopy }\end{array}$ \\
$\begin{array}{l}\text { Urinalysis with reflex to } \\
\text { culture }\end{array}$ & $\begin{array}{l}\text { Perform urine dipstick, if positive } \\
\text { for protein>trace, any blood, nitrite } \\
\text { or leukocyte esterase then reflex to } \\
\text { microscopy and urine culture }\end{array}$ \\
Urine macroscopic & Macroscopic dipstick urinalysis only \\
Urine microscopic & Urine sediment examination only \\
\hline
\end{tabular}

'frequently ordered' order set within a computerised physician order entry system (CPOE). We then evaluated the impact of this change on urine testing practices in the ED of an urban, academic medical centre.

\section{METHODS}

\section{Study design and setting}

We conducted a before-after study of all patients seen at our hospital ED between 1 September 2015 and 30 November 2015. This ED averages approximately 93000 patient visits annually.

\section{Electronic order sets/intervention}

Urine order sets at our hospital ED are commonly ordered from the drop-down menu of highly accessible 'frequently ordered tests' in the CPOE system. The urine order sets available in 'frequently ordered tests' and the corresponding definitions are illustrated in table 1. On 21 October 2015, a quality improvement project was implemented to reduce unnecessary urine cultures. The intervention consisted of removing the orders for 'urinalysis with reflex to culture', 'urine macroscopic' and 'urine microscopic' from this highly accessible list of 'frequently ordered tests' in the CPOE system, while retaining only 'urinalysis with reflex to microscopy' (table 2).

During the entire study period, 'urinalysis with reflex to microscopy', 'urinalysis with reflex to culture', 'urine macroscopic' and 'urine microscopic' were available through a specialised list of 'haematology/coagulation/urinalysis and microbiology' orders requiring two additional mouse clicks and an order for 'urine culture' alone was available in the 'microbiology' order set requiring three additional mouse clicks to access. ED attending and resident physicians were educated about this change through in-person education and electronic correspondence. No additional interventions were implemented during the study period related to urine culture order sets.

\section{Data collection}

Patient and laboratory data were abstracted from the hospital electronic medical informatics database. Data included ED patient demographics, laboratory test results (urinalysis, microscopic exam and urine culture)
Table 2 Changes in the urine order sets within study emergency department

\begin{tabular}{|c|c|c|}
\hline Category & Pre intervention & Post intervention \\
\hline \multicolumn{3}{|l|}{ Frequent labs } \\
\hline & $\begin{array}{l}\text { Urinalysis with reflex to } \\
\text { microscopy }\end{array}$ & $\begin{array}{l}\text { Urinalysis with reflex } \\
\text { to microscopy }\end{array}$ \\
\hline & $\begin{array}{l}\text { Urinalysis with reflex to } \\
\text { culture }\end{array}$ & \\
\hline & Urine macroscopic & \\
\hline & Urine microscopic & \\
\hline \multicolumn{3}{|c|}{$\begin{array}{l}\text { Haematology / } \\
\text { coagulation/ } \\
\text { urinalysis } \\
\text { (required two additional } \\
\text { mouse clicks) }\end{array}$} \\
\hline & $\begin{array}{l}\text { Urinalysis with reflex to } \\
\text { microscopy }\end{array}$ & $\begin{array}{l}\text { Urinalysis with reflex } \\
\text { to microscopy }\end{array}$ \\
\hline & $\begin{array}{l}\text { Urinalysis with reflex to } \\
\text { culture }\end{array}$ & $\begin{array}{l}\text { Urinalysis with reflex } \\
\text { to culture }\end{array}$ \\
\hline & Urine macroscopic & Urine macroscopic \\
\hline & Urine microscopic & Urine microscopic \\
\hline \multicolumn{3}{|c|}{$\begin{array}{l}\text { Microbiology } \\
\text { (required three } \\
\text { additional mouse clicks) }\end{array}$} \\
\hline & Urine culture & Urine culture \\
\hline
\end{tabular}

and disposition. The medical informatics database does not include orders, only tests results. For urine cultures with accompanying urinalysis or microscopy, the time between the culture and urinalysis and/or microscopy was calculated. The presence of an indwelling urinary catheter was identified based on specimen type indicated by the ordering clinician. For patients with multiple urine tests, we treated each sample as an independent observation. Urine cultures that were no growth or contaminated were considered negative for this analysis. Any growth $\geq 50000$ colonies $/ \mathrm{ml}$ for clean catch specimen and $\geq 5000$ colonies $/ \mathrm{ml}$ for catheterised specimen are treated as positive urine culture results. Leukocyte esterase $\geq 1$ identified on urinalysis and $>5$ WBCs per high-power field on urine microscopy were treated as abnormal/positive test results.

\section{Statistical analysis}

We compared patient and urine testing data during the 50 days preceding and 41 days following the intervention (Wilcoxon rank-sum test, $\chi^{2}$ or univariable logistic regression where appropriate). Demographic characteristics were compared only for patients with $\geq 1$ urine test (ie, urine culture, urinalysis and microscopy) ordered during their ED visit, whereas urine testing rates (per $1000 \mathrm{ED}$ patient visits) were calculated for entire study period, pre intervention and post intervention period using the ED visits as the denominator. Regression modelling with autoregressive integrated moving average (ARIMA) errors was used to test the significance of the percentage change in the daily urine 
Table 3 Comparison of 6499 patients who had any urine testing in the emergency department (ED) before and after intervention

\begin{tabular}{|c|c|c|c|c|}
\hline & Study cohort* & $\begin{array}{l}\text { Pre intervention } \\
\text { (1 September to } 20 \\
\text { October) } \\
\end{array}$ & $\begin{array}{l}\text { Post intervention } \\
\text { ( } 21 \text { October to } 30 \\
\text { November) }\end{array}$ & \\
\hline & $\mathrm{n}=6499$ & $\mathrm{n}=3711$ & $\mathrm{n}=2788$ & $P$ value \\
\hline \multicolumn{5}{|l|}{ Patient characteristics } \\
\hline Age, median (IQR) & $46(30-62)$ & $46(29-62)$ & $46(30-62)$ & 0.88 \\
\hline \multicolumn{5}{|l|}{ Race, $n(\%)$} \\
\hline White & $2499(38.5)$ & $1453(39.2)$ & $1046(37.5)$ & Ref \\
\hline Black & $3687(56.7)$ & $2086(56.2)$ & $1601(57.4)$ & 0.22 \\
\hline Other & $313(4.8)$ & $172(4.6)$ & $141(5.1)$ & 0.28 \\
\hline \multicolumn{5}{|l|}{ Sex, n (\%) } \\
\hline Male & $2441(37.6)$ & $1406(37.9)$ & $1035(37.1)$ & 0.53 \\
\hline Female & $4058(62.4)$ & $2305(62.1)$ & $1753(62.9)$ & Ref \\
\hline \multicolumn{5}{|l|}{ Discharge status, n (\%) } \\
\hline Admitted to hospital & $2916(44.9)$ & $1625(43.8)$ & $1291(46.3)$ & 0.55 \\
\hline Discharged to home from ED & $3314(51.0)$ & $1931(52.0)$ & $1383(49.9)$ & 0.84 \\
\hline Discharged to other facility/expired/AMA $†$ & $269(4.1)$ & $155(4.2)$ & $114(4.1)$ & Ref \\
\hline \multicolumn{5}{|l|}{ Urine testing practices } \\
\hline Urinalysis, $n$ (per 1000 ED visits) & $6556(285.7)$ & $3744(291.5)$ & $2812(278.4)$ & 0.03 \\
\hline Leukocyte esterase positive, n (\%) & $2553(38.9)$ & $1428(38.1)$ & $1125(40.0)$ & 0.08 \\
\hline Urine microscopy, n (per 1000 ED visits) & $4408(192.1)$ & $2524(196.5)$ & $1813(179.5)$ & 0.001 \\
\hline \multicolumn{5}{|l|}{ Urine microscopy result (WBC/hpf), n (\%) } \\
\hline$\leq 5$ & $2540(57.6)$ & $1500(59.4)$ & $1040(55.2)$ & Ref \\
\hline$>5-49$ & $1209(27.4)$ & $675(26.8)$ & $534(28.3)$ & 0.06 \\
\hline$\geq 50$ & $659(15.0)$ & $349(13.8)$ & $310(16.5)$ & 0.005 \\
\hline Urine cultures performed, $\mathrm{n}$ (per 1000 ED visits) & $997(43.4)$ & $697(54.3)$ & $300(29.7)$ & $<0.0001$ \\
\hline Positive culture, $\mathrm{n}(\%)$ & $392(39.3)$ & $270(38.7)$ & $122(40.7)$ & 0.43 \\
\hline Catheterised cultures, n (per 1000 ED visits) & $49(2.1)$ & $22(1.7)$ & $27(2.7)$ & 0.0002 \\
\hline Isolated culture, $\mathrm{n}(\%) \ddagger$ & $25(2.5)$ & $16(2.3)$ & $9(3.0)$ & 0.51 \\
\hline
\end{tabular}

tests ordered per 1000 ED patient visits, adjusting for temporal trends in the data. Data were analysed using SAS V.9.3 (SAS Institute).

\section{RESULTS}

\section{Patient characteristics}

During the study period, $6499(28.2 \%)$ of 22948 ED patients had $\geq 1$ urine test ordered (3711/10 102 (28.9\%) pre change vs 2788/12846 (27.6\%) post change; $\mathrm{P}=0.03)$. Median age of all patients with $\geq 1$ urine test ordered was 46 years (IQR 30-62). Approximately 57\% of patients were black and $62.4 \%$ were female. There were no differences in gender, race or discharge status pre intervention versus post intervention (table 3 ).

\section{Urine testing}

Urine testing rates decreased in the post intervention period for urinalysis (291.5 pre intervention vs 278.4 per $1000 \mathrm{ED}$ visits post intervention, $\mathrm{P}=0.03$ ), urine microscopy (196.5 vs $179.5, \mathrm{P}=0.001)$ and urine culture $(54.3$ vs $29.7, \mathrm{P}<0.001)$. Post intervention, there was no change in the proportion of positive urinalyses (1428/3744 (38.1\%) pre intervention vs $1125 / 2812(40.0 \%)$ post intervention; $\mathrm{P}=0.08)$ or urine cultures $(270 / 697(38.7 \%)$ pre intervention vs $122 / 300(40.7 \%)$ post intervention; $\mathrm{P}=0.43$ ) (figure 1). When adjusted for overall temporal trend, the daily culture rate per $1000 \mathrm{ED}$ visits decreased by $46.6 \%$ ( $-46.6 \%, 95 \%$ CI $-66.2 \%$ to $-15.6 \%)$, but urinalysis $(0.4 \%, 95 \% \mathrm{CI}-30.1 \%$ to $44.4 \%)$, microscopy $(-6.5 \%, 95 \% \mathrm{CI}-36.0 \%$ to $36.6 \%)$ and catheterised urine culture rates $(17.9 \%, 95 \% \mathrm{CI}-16.9$ to 67.4) were unchanged.

\section{Type of urine cultures ordered}

Since we could not directly assess if urine reflex testing was ordered, we approximated the type of urine cultures ordered (ie, urinalysis with reflex to culture vs other) using the specimen collection time 


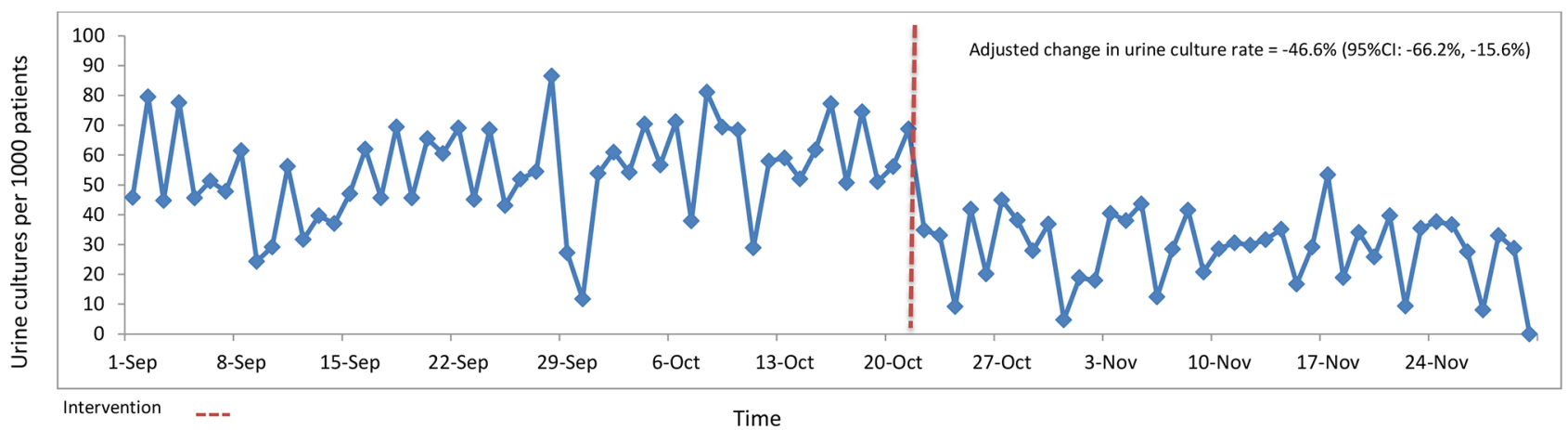

Figure 1 Urine culturing practices in emergency department (1 September 2015 to 30 November 2015). Adjusted for overall temporal trend on autoregressive integrated moving average.

for urinalysis/urine microscopy compared with urine culture collection time. We assumed that urine tests with simultaneous collection times to represent reflex testing. A review of the patient charts from a small, random sample of cultures collected within $10 \mathrm{~min}$ of urinalysis/urinemicroscopy group found these to be 'urinalysis with reflex to culture' orders (data not shown). As shown in table 4, tests per 1000 ED visits for all types of urine cultures decreased significantly post intervention $(\mathrm{P}<0.05)$ except for urine cultures obtained $>10$ min after urinalysis/urine microscopy.

\section{Subsequent urine culture testing in hospital}

To determine if an admitting service felt a urine culture was 'missed' in the course of ED care, we evaluated patients subsequently admitted to hospital who had either urinalysis or microscopy test obtained in the $\mathrm{ED}$, but no urine culture $(n=2375)$. There was no significant change in the proportion of individuals who had a urine culture ordered in the hospital within 24 hours of an ED urinalysis and/or microscopy (64/1272 (5.0\%) pre intervention vs $75 / 1103(6.8 \%)$ post intervention; $\mathrm{P}=0.07)$ or at any time during hospitalisation (121/1272 (9.5\%) pre intervention vs $131 / 1103(11.8 \%)$ post intervention; $\mathrm{P}=0.07)$.

\section{DISCUSSION}

We found that removal of certain urine testing orders from the frequently ordered test window in the ED CPOE system was associated with a $46.6 \%$ reduction in urine cultures ordered in the ED, without significant changes in the frequency of urinalysis and microscopy tests ordered, and the proportion of the positive urine cultures, after accounting for temporal trends.

The ED is one of the most common locations for urine testing, yet previous efforts to reduce unnecessary urine cultures, including education, have had limited success. ${ }^{6-8}$ In a recent study by Stagg et $a l,{ }^{4}$ institution of a two-step model for urine culture ordering resulted in a decrease in the number of unnecessary urine cultures processed, a decrease in patient callbacks for positive results and improved antimicrobial prescribing for UTI. Step 1 included an order for urine analysis and urine culture, and step 2 included processing the urine for culture and susceptibility (if required) after an ED physician assessment. Healthcare providers were educated about the intervention and the goal of reducing urine culture rates. However, the authors reported no significant decrease in the total number of urine cultures ordered in the intervention period. Jones and colleagues demonstrated that a reflex urine culture cancellation protocol in the ED, based on low-risk findings for infection on urinalysis, would have eliminated 39\% of unnecessary cultures. ${ }^{1}$ Although this reflex urine culture cancellation protocol was externally validated by Hertz et $a l,{ }^{9}$ these were mainly derivative studies using retrospective data and were not prospectively implemented. While stewardship efforts ${ }^{5}$ reduce unnecessary antibiotic prescriptions and the associated costs, they are time-intensive interventions and

Table 4 Type of urine cultures ordered, based on collection time

\begin{tabular}{|c|c|c|c|c|c|}
\hline & \multicolumn{2}{|c|}{ Pre intervention } & \multicolumn{2}{|c|}{ Post intervention } & \multirow[b]{2}{*}{$P$ value } \\
\hline & $\mathrm{N}$ & Tests per 1000 ED visits & $\mathrm{N}$ & Tests per 1000 ED visits & \\
\hline $\begin{array}{l}\text { Simultaneous urine culture and urinalysis collection times (approximating } \\
\text { urinalysis with reflex to culture) }\end{array}$ & 264 & 20.6 & 99 & 9.8 & $<0.0001$ \\
\hline Urine cultures collected $<10 \mathrm{~min}$ of urinalysis/urine microscopy & 242 & 18.8 & 62 & 6.1 & $<0.0001$ \\
\hline $\begin{array}{l}\text { Urine cultures obtained }>10 \mathrm{~min} \text { after urinalysis/urine microscopy and urine } \\
\text { cultures without any urinalysis/urine microscopy }\end{array}$ & 191 & 14.9 & 139 & 13.8 & 0.484 \\
\hline Overall & 697 & 54.3 & 300 & 29.7 & $<0.0001$ \\
\hline
\end{tabular}

$E D$, emergency department. 
require constant monitoring. The effect of removing multiple urine testing orders from a frequently ordered test set on ED physician ordering practices has not been previously well-described.

Our intervention showed that retaining only 'urinalysis with reflex to microscopy' and removing all other urine tests from a list of frequently ordered tests in the ED CPOE led to a $46.6 \%$ reduction in urine cultures ordered in the ED. We also evaluated for possible shift of urine culture testing to in-hospital locations among admitted patients and found no significant change. By removing these order sets, ED physicians were made to order the urine cultures alone through deeply embedded order sets which required three additional mouse clicks. Relatively simple changes in CPOE preferences can greatly alter clinician ordering practices. Olson et al reported that preselecting a post-transfusion haematocrit order within a red cell transfusion order set increased frequency of testing from $8.3 \%$ to $57.4 \%$ after transfusion. ${ }^{10}$ Similar findings were observed for CPOE use in managing congestive heart failure, ${ }^{11}$ analgesic treatment for renal colic ${ }^{12}$ and sepsis. ${ }^{13}$ According to the Medicare Clinical Laboratory Fee Schedule, our intervention resulted in savings of $\$ 5955$ during the study period ( $\$ 10455$ in the pre-period vs $\$ 4500$ in the post intervention, at national median Medicare payment rate of $\$ 15.00$ per urine culture). ${ }^{14}$

We focused mainly on the 'urinalysis with reflex to culture' as it is a standard order set across many institutions which avoids recollection of urine. ED physician and resident education may have influenced the fewer urine culture orders in the post intervention period, but this was unlikely as the education was mainly notification of the removal order sets and not about reducing urine culture rates. Though we noticed a slight drop in the urinalysis and urine microscopy rates post intervention, this was not statistically significant when adjusted for temporal trends using ARIMA modelling. The slight drop in the rates may be due to the removal of 'urine macroscopic' and 'urine microscopic' from the list of 'frequently ordered tests'. Also there might have been a change in the ED patient population over time due to this study occurring over the start of influenza season in our region. ${ }^{15}$

Limitations of our study include retrospective design, absence of chart review and generalisability of results because of single-centre study setting. Due to the nature of the laboratory database in our hospital, we were unable to get the actual frequency of urinalysis with reflex to culture versus other ways of ordering the urine cultures directly from the database. However, we used the collection time to determine if the urine cultures ordered were part of the urinalysis with reflex to culture versus other ways of ordering. We also did not have data on antibiotic use, limiting the assessment of positive urine cultures for antimicrobial therapy directed against ASB. Although there was no significant change in the proportion of individuals who had a urine culture ordered in the hospital within
24 hours of an ED urinalysis and/or microscopy, our study may be underpowered to detect a change. Strengths of our study include a large sample size and the use of an automated electronic intervention that can be easily replicated at other institutions employing CPOE. We also adjusted for overall temporal trends to account for autocorrelation due to patient mix and influenza season.

Results of our study complement recent intervention studies to improve urine culture practices, such as two-step urine culture ordering, ${ }^{4}$ reflex urine culture cancellation protocol, ${ }^{19}$ pharmacist-driven antimicrobial stewardship efforts, ${ }^{5}$ distribution of a pocket card to clinicians outlining a diagnostic algorithm for UTI diagnosis with case-based audit and feedback ${ }^{2}$ and suppression of urine culture results from non-catheterised inpatients. ${ }^{16}$

In conclusion, we found that retaining only 'urinalysis with reflex to microscopy' and removing all other urine tests from a frequently ordered electronic order set resulted in a $46.6 \%$ reduction in urine cultures obtained in the ED. This supports the hypothesis that minor changes in CPOE design can have a significant impact on physician ordering practices. Further studies are needed to evaluate the role of CPOE across the spectrum of care to reduce excessive urine culture testing and prevent unnecessary antimicrobial use.

Contributors All authors made substantial contribution to the project conception, study design, data analysis and drafting of the paper. All authors have approved the final version of the paper for publication.

Funding This study was funded by Centers for Disease Control and Prevention Epicenters Program (grant no. 1 U54CK000482-01) to David K. Warren.

Competing interests None declared.

Provenance and peer review Not commissioned; externally peer reviewed.

(C) Article author(s) (or their employer(s) unless otherwise stated in the text of the article) 2018. All rights reserved. No commercial use is permitted unless otherwise expressly granted.

\section{REFERENCES}

1 Jones CW, Culbreath KD, Mehrotra A, et al. Reflect urine culture cancellation in the emergency department. J Emerg Med 2014;46:71-6.

2 Trautner BW, Grigoryan L, Petersen NJ, et al. Effectiveness of an antimicrobial stewardship approach for urinary catheterassociated asymptomatic bacteriuria. JAMA Intern Med 2015;175:1120-7.

3 Nicolle LE, Bradley S, Colgan R, et al. Infectious diseases society of America guidelines for the diagnosis and treatment of asymptomatic bacteriuria in adults. Clin Infect Dis 2005;40:643-54.

4 Stagg A, Lutz H, Kirpalaney S, et al. Impact of two-step urine culture ordering in the emergency department: a time series analysis. BMJ Qual Saf 2018;27:140-7.

5 Zhang X, Rowan N, Pflugeisen BM, et al. Urine culture guided antibiotic interventions: a pharmacist driven antimicrobial stewardship effort in the ED. Am J Emerg Med 2017;35:594-8. 
6 Loeb M, Brazil K, Lohfeld L, et al. Effect of a multifaceted intervention on number of antimicrobial prescriptions for suspected urinary tract infections in residents of nursing homes: cluster randomised controlled trial. BMJ 2005;331:669.

7 Silver SA, Baillie L, Simor AE. Positive urine cultures: a major cause of inappropriate antimicrobial use in hospitals? Can J Infect Dis Med Microbiol 2009;20:107-11.

8 Wilson ML, Gaido L. Laboratory diagnosis of urinary tract infections in adult patients. Clin Infect Dis 2004;38:1150-8.

9 Hertz JT, Lescallette RD, Barrett TW, et al. External validation of an ED protocol for reflex urine culture cancelation. Am J Emerg Med 2015;33:1838-9.

10 Olson J, Hollenbeak C, Donaldson K, et al. Default settings of computerized physician order entry system order sets drive ordering habits. J Pathol Inform 2015;6:16.

11 Reingold S, Kulstad E. Impact of human factor design on the use of order sets in the treatment of congestive heart failure. Acad Emerg Med 2007;14:1097-105.
12 Netherton SJ, Lonergan K, Wang D, et al. Computerized physician order entry and decision support improves ED analgesic ordering for renal colic. Am J Emerg Med 2014;32:958-961.

13 Winterbottom F, Seoane L, Sundell E, et al. Improving sepsis outcomes for acutely ill adults using interdisciplinary order sets. Clin Nurse Spec 2011;25:180-5.

14 Centers for Medicare and Medicaid Services. Clinical laboratory fee schedule. 2017 https://www.cms.gov/Medicare/ Medicare-Fee-for-Service-Payment/ClinicalLabFeeSched/ Clinical-Laboratory-Fee-Schedule-Files-Items/17CLAB.html? DLPage $=1 \&$ DLEntries $=100 \&$ DLSort $=2 \&$ DLSortDir $=$ descending (accessed 01 Dec 2017).

15 St. Louis County. Summary of Flu Activity 2015 - 2016. Missouri: St. Louis County, 2017.

16 Leis JA, Rebick GW, Daneman N, et al. Reducing antimicrobial therapy for asymptomatic bacteriuria among noncatheterized inpatients: a proof-of-concept study. Clin Infect Dis 2014;58:980-3. 